\title{
Primary A (H1N1) pdm09 Influenza Pneumonia Diagnosed on Reverse Transcription-polymerase Chain Reaction (RT-PCR) of Bronchoalveolar Lavage Fluid but not Rapid Tests with Nasopharyngeal Swabs
}

\author{
Noriyuki Ohkura, Mayuko Tani, Masaru Nishitsuji and Koichi Nishi
}

\begin{abstract}
A 47-year-old man with a fever was highly suspected of having influenza A infection since his wife and son who lived with him had been diagnosed with influenza A. Although repeated rapid tests with a nasopharyngeal swab showed negative findings, the patient developed bilateral pneumonia and reverse transcription polymerase chain reaction (PCR) for A (H1N1) pdm09 virus in the bronchoalveolar lavage fluid was positive. We therefore diagnosed him with primary influenza pneumonia and initiated treatment with peramivir plus corticosteroids, which rapidly improved his condition. During the influenza season, sample collection from the lower airway and PCR should be considered for the definitive diagnosis of primary influenza viral pneumonia.
\end{abstract}

Key words: A (H1N1) pdm09, PCR, primary influenza pneumonia

(Intern Med 54: 1441-1445, 2015)

(DOI: 10.2169/internalmedicine.54.3823)

\section{Introduction}

A pandemic caused by A (H1N1) pdm09 influenza virus occurred during the 2009/2010 seasons. Infection with A (H1N1) pdm09 influenza virus results in a broad spectrum of clinical syndromes, ranging from afebrile upper respiratory illnesses to fulminant viral pneumonia. Dyspnea, tachypnea, chest pain, hemoptysis or purulent sputum, prolonged or recurrent bouts of fever, an altered mental status, manifestations of dehydration and the reappearance of lower respiratory tract symptoms after improvement are signs of progression to more severe disease and/or complications. The principal clinical syndrome leading to hospitalization and intensive care is diffuse viral pneumonitis associated with severe hypoxemia, acute respiratory distress syndrome (ARDS) (1). Many reports have described the clinical diagnosis of A (H1N1) pdm09 influenza virus. Although nasopharyngeal aspirates or swabs collected early after the onset of symptoms provide suitable samples, endotracheal or bronchoscopic aspirates have higher yields in patients with lower respiratory tract illnesses $(2,3)$. The sensitivity of rapid tests (immunofluorescent antigen assays) with nasopharyngeal swabs is low in cases of A (H1N1) pdm09 infection $(2,4,5)$, and repeated collection of multiple respiratory specimens is recommended in the patients with a high clinical suspicion of $\mathrm{H} 1 \mathrm{~N} 1$ infection (1). We herein report the case of a 47-year-old man with primary A (H1N1) pdm09 influenza viral pneumonia diagnosed on reverse transcription polymerase chain reaction (RT-PCR) of the bronchoalveolar lavage fluid (BALF) but not rapid tests with nasopharyngeal swabs.

\section{Case Report}

A 47-year-old man complained of fever and headache at a nearby clinic in January 2014. His wife and son who lived with him also exhibited a fever during the same time period and were diagnosed with influenza A using rapid tests with nasopharyngeal swabs. Three rapid tests with nasopharyngeal swabs were performed at the clinic on three different days; however, all results were negative. While antibiotics 

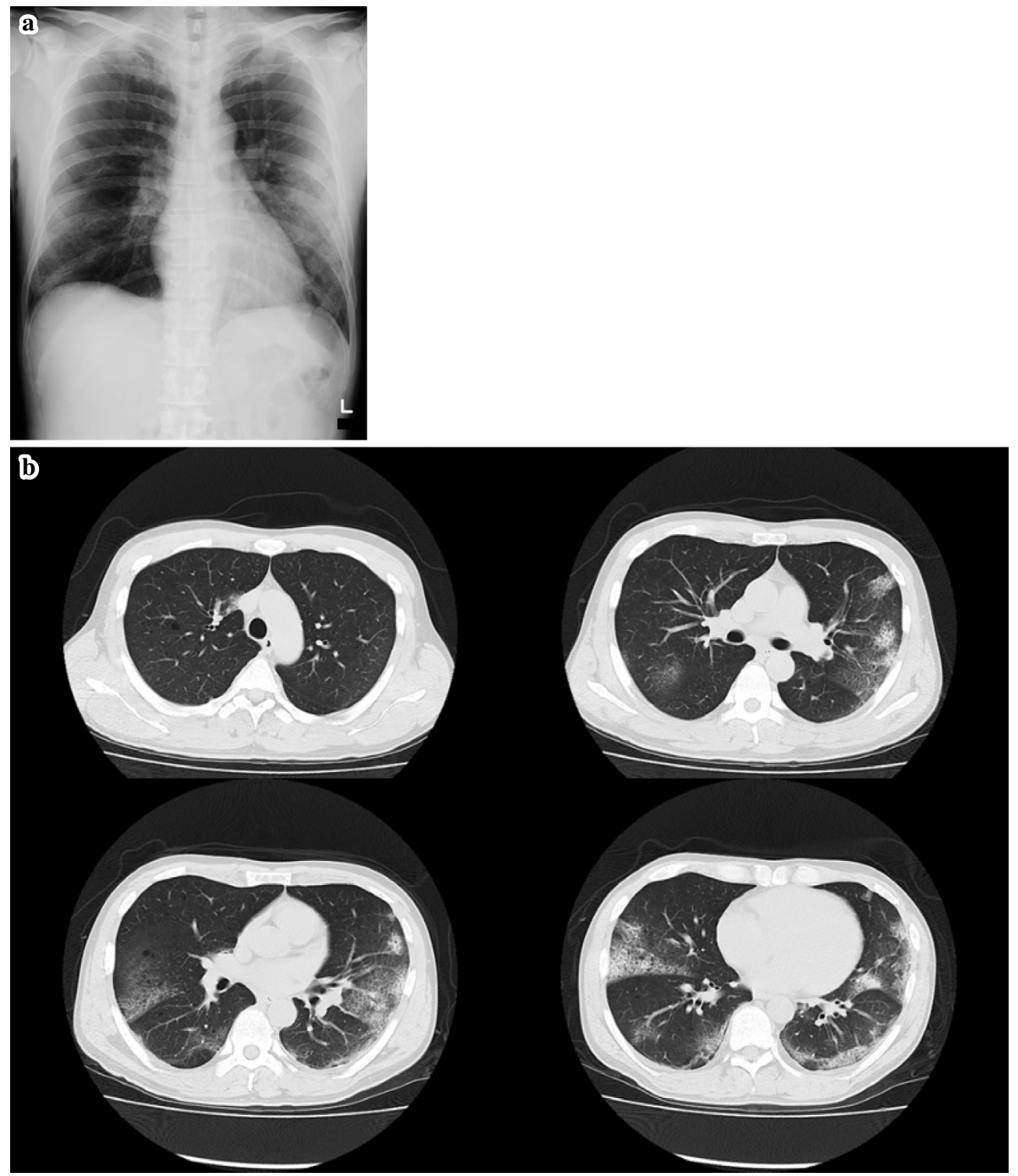

Figure 1. Chest imaging findings on admission. A chest X-ray showed bilateral patchy opacity (a). Chest computed tomography demonstrated ground glass opacity, a crazy-paving pattern and consolidation in the bilateral lung fields (b).

(cefcapene pivoxil and azithromycin) were prescribed, the patient's symptoms persisted for seven days. Since a chest X-ray showed consolidation in both lungs (Fig. 1a), he was referred to our hospital. He had no significant underlying diseases, although he had smoked from 20 to 47 years of age. He did not receive the influenza vaccine during the relevant season. On admission, his respiratory rate was 20 breaths per minute, with a heart rate of 75 beats per minute, blood pressure of $97 / 56 \mathrm{mmHg}$ and percutaneous oxygen saturation of $96 \%$. Chest auscultation revealed inspiratory fine crackles on the left side, and chest computed tomography (CT) showed bilateral ground-glass opacity, a crazypaving pattern and consolidation (Fig. 1b). The laboratory data disclosed a white blood cell count of $3,480 / \mu \mathrm{L}$ (neutrophils: $73.8 \%$, lymphocytes: $16.7 \%$, eosinophils: $0 \%$, monocytes: $8.9 \%$, basophils: $0.6 \%$ ), hemoglobin level of $13.0 \mathrm{~g} /$ $\mathrm{dL}$, platelet count of $17.7 \times 10^{4} / \mu \mathrm{L}$, total protein level of 7.0 $\mathrm{g} / \mathrm{dL}$, urea nitrogen level of $13.1 \mathrm{mg} / \mathrm{dL}$, creatinine level of $0.7 \mathrm{mg} / \mathrm{dL}$, lactic dehydrogenase level of $410 \mathrm{IU} / \mathrm{L}$, total bilirubin level of $0.2 \mathrm{mg} / \mathrm{dL}$, aspartate aminotransferase (AST) level of $121 \mathrm{U} / \mathrm{L}$, alanine aminotransferase (ALT) level of $65 \mathrm{U} / \mathrm{L}$ and C-reactive protein (CRP) level of 6.2 $\mathrm{mg} / \mathrm{dL}$. Urinary antigen tests for both Legionella pneumophila and Streptococcus pneumoniae were negative, and a rapid influenza virus test (ImunoAce, TAUNS, Numazu, Japan) with a nasopharyngeal swab was negative. In addition, sputum smears and culture were negative for bacteria, including acid-fast bacteria, and the results of isothermal loopmediated amplification of Legionella pneumophila and Mycoplasma pneumonia in the sputum were negative. In contrast, fiberoptic bronchoscopy performed on the second hospital day showed abnormal findings, including inflammation of the bronchial mucosa in the bronchial lumen. A total of $53 \mathrm{~mL} / 150 \mathrm{~mL}$ of BALF recovered from the left superior 


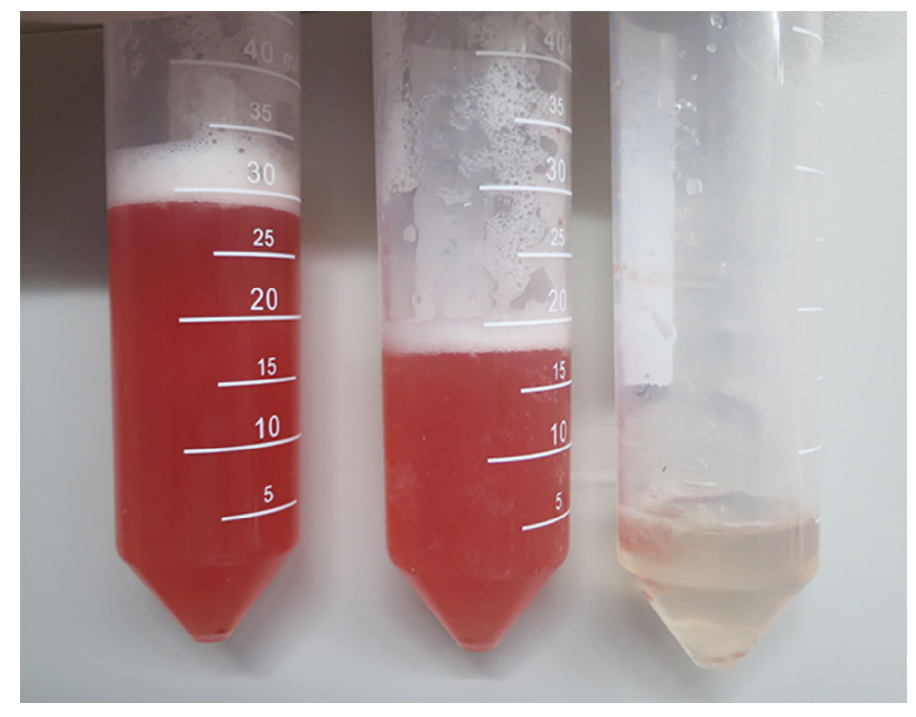

Figure 2. Appearance of the bronchoalveolar lavage fluid (BALF). Bronchoscopy performed on the second hospital day. BALF from the left superior lingular region displayed hemorrhagic features on sequential samples.

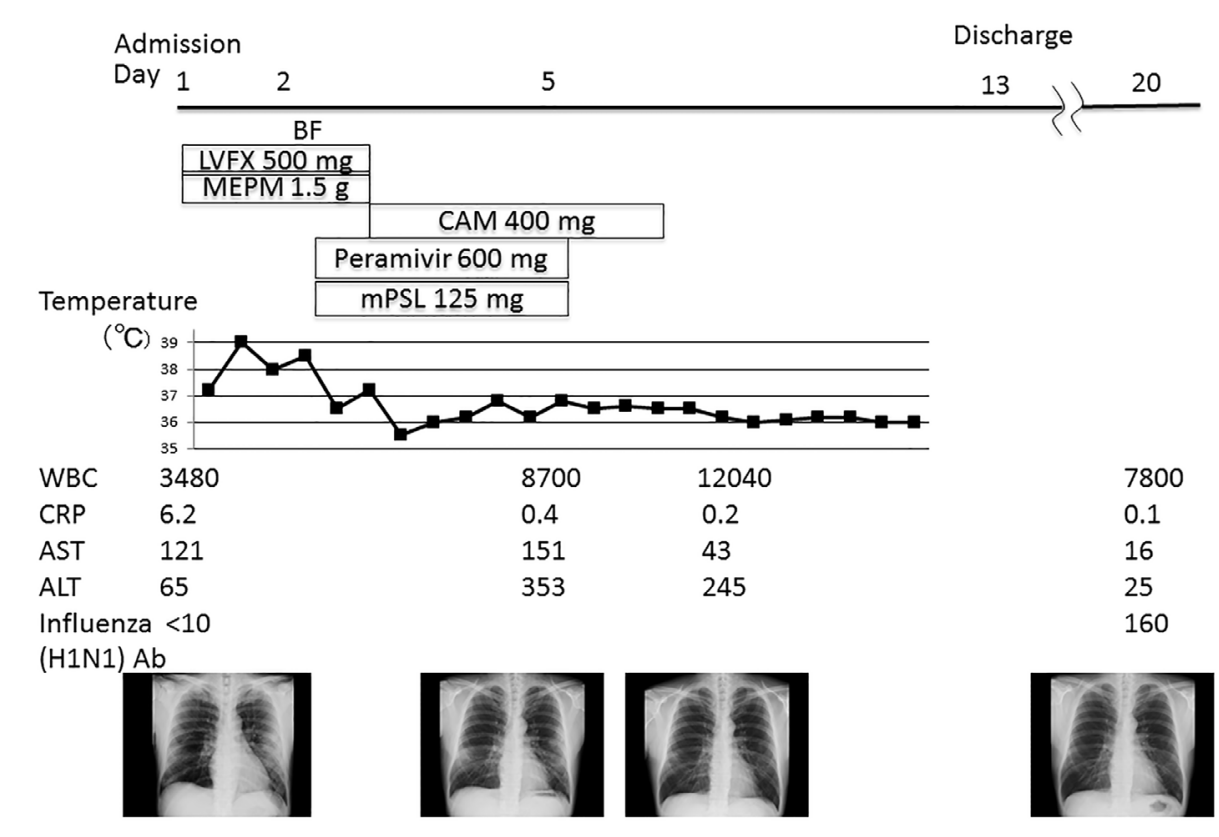

Figure 3. Clinical course. The patient's clinical course showed improvements in the fever, radiological findings, inflammatory response and hepatopathy following the administration of a neuraminidase inhibitor and corticosteroids. The patient was discharged on hospital day 13. Three weeks after admission, the serum antibody titer for influenza H1N1 had increased significantly. Abbreviations: ALT: alanine aminotransferase, AST: aspartate aminotransferase, CAM: clarithromycin, CRP: Creactive protein, LVFX: levofloxacin, MEPM: meropenem, mPSL: methylprednisolone, WBC: white blood cell

lingular region disclosed hemorrhagic features on sequential samples (Fig. 2). The white blood cell count in the BALF was $5.3 \times 10^{5}$ cells $/ \mathrm{mL}$ (macrophages: $44.3 \%$, lymphocytes: $55.7 \%$, neutrophils: $0 \%$, eosinophils: $0 \%$ ), although a BALF smear and culture for bacteria, including acid-fast bacteria, were negative. While the findings of RT-PCR for the influenza virus with a nasopharyngeal swab and rapid test using BALF were negative, RT-PCR with BALF was positive for the A (H1N1) pdm09 influenza virus. We therefore diagnosed the patient with primary influenza viral pneumonia and initiated therapy with an intravenous neuraminidase inhibitor, peramivir (600 mg/day), plus corticosteroids (mPSL: $125 \mathrm{mg} / \mathrm{day}$ ) for three days. Consequently, the patient's fever, CRP, AST, and ALT levels and consolidation on chest $\mathrm{X}$-rays improved, and he was discharged on hospital day 13 . Three weeks after admission, the serum hemagglutinin (HA) 
antibody titer for influenza H1N1 had increased from $<10$ to 160 (Fig. 3).

\section{Discussion}

As a characteristic in the present case, PCR of a sample obtained from the lower airway (BALF) was positive for the A (H1N1) pdm09 influenza virus. However, rapid tests (immunofluorescent antigen assays) and PCR of samples from the upper airway were negative, as was a rapid test of a sample from the lower airway. The type of assay and the origin of the sample may affect the accuracy of diagnosis of A (H1N1) pdm09 influenza virus infection (2). Indeed, it has been reported that nasopharyngeal aspirates or swabs collected early after the onset of symptoms provide suitable samples, whereas endotracheal or bronchoscopic aspirates have higher yields in patients with lower respiratory tract illnesses $(2,3)$. According to a previous report, $19 \%$ of patients with detectable A (H1N1) pdm09 viral RNA in bronchoscopic samples had negative upper respiratory tract samples. Furthermore, direct or indirect immunofluorescence tests are less sensitive than RT-PCR (2). The efficacy of several commercial influenza rapid tests for assessment of the upper airway has been evaluated in case of A (H1N1) pdm09, demonstrating low sensitivity for the detection of A (H1N1) pdm09 with a sensitivity and specificity of $48.7 \%$ and $96.5 \%$ (4) versus, $47 \%$ and $95 \%$ for PCR analyses (5), respectively. Negative results for single respiratory specimens do not rule out the possibility of A (H1N1) pdm09 influenza virus infection, and repeated collection of multiple types of respiratory specimens is recommended if clinical suspicion is high (1).

The findings of ground glass opacity, a crazy-paving pattern and consolidation in the bilateral lung fields on CT observed in the present case are consistent with those of primary viral pneumonia reported previously (6). However, these findings on CT are not specific to A (H1N1) pdm09 influenza viral pneumonia. Nevertheless, the potential for influenza viral pneumonia should be taken into consideration in the differential diagnosis, especially during the pandemic season.

In a report of lung autopsy findings of A (H1N1) pdm09 influenza virus pneumonia (7), pathological changes associated with H1N1 infection were localized to the lungs, in which three distinct histological patterns could be identified: diffuse alveolar damage (DAD), necrotizing bronchiolitis and DAD with intense alveolar hemorrhage. In addition to the presence of infectious cells in the upper respiratory and tracheobronchial epithelium and mucosal glands, the A (H1N1) pdm09 influenza virus targets cells of the alveolar lining (type I and II pneumocytes) (8). Clinically, one of the most frequent causes of diffuse alveolar hemorrhage in cases of infectious disease is A (H1N1) influenza virus (9). The features of hemorrhagic BALF noted in the present case were consistent with the pathological findings of A (H1N1) pdm09 influenza virus infection.
The present patient improved with adjunctive corticosteroid therapy. Corticosteroids possess anti-inflammatory properties and are known to modulate inflammation, suppress the immune system and regulate the stress response. Their use in patients with influenza infections has only been evaluated in retrospective studies, with controversial results. In ARDS patients, with and without confirmed H1N1 influenza infection, prolonged low- to moderate-dose corticosteroid treatment is well tolerated and associated with significant improvements in scores for lung injury and multiple organ dysfunction as well as low hospital mortality (10). In contrast, in a retrospective cohort study of hospitalized patients with A (H1N1) pdm09 influenza virus infection in China, the early use (less than 72 hours after onset) of glucocorticoids for fever reduction and pneumonia prevention was found to be associated with the subsequent development of critical illnesses, even after adjusting for the presence of underlying diseases or risk factors (hazard ratio: 2.9, 95\% CI: 1.3-6.2) (11). Further research is necessary to improve patient selection and facilitate the identification of subgroups that may benefit from adjunctive steroid therapy.

During the influenza pandemic season, sample collection from the lower airway and PCR should be performed to obtain a definitive diagnosis of primary influenza viral pneumonia, even if rapid tests (immunofluorescent antigen assays) with nasopharyngeal swabs are negative.

The authors state that they have no Conflict of Interest (COI).

\section{Acknowledgement}

We gratefully acknowledge the staff of the Ishikawa prefectural Institute of Public Health and Environmental Science for their kind assistance and technical help.

\section{References}

1. Writing Committee of the WHO Consultation on Clinical Aspects of Pandemic ( H 1 N 1) 2009 Influenza, Bautista E, Chotpitayasunondh T, Gao Z, et al. Clinical aspects of pandemic 2009 influenza A (H1N1) virus infection. N Engl J Med 362: 1708-1719, 2010.

2. Blyth CC, Iredell JR, Dwyer DE. Rapid-test sensitivity for novel swine-origin influenza A (H1N1) virus in humans. N Engl J Med 361: 2493, 2009.

3. Fleury H, Burrel S, Balick Weber C, et al. Prolonged shedding of influenza $\mathrm{A}(\mathrm{H} 1 \mathrm{~N} 1) \mathrm{v}$ virus: two case reports from France 2009. Euro Surveill 14: 2009.

4. Karre T, Maguire HF, Butcher D, Graepler A, Weed D, Wilson ML. Comparison of Becton Dickinson Directigen EZ Flu A+B test against the CDC real-time PCR assay for detection of 2009 pandemic influenza A/H1N1 virus. J Clin Microbiol 48: 343-344, 2010.

5. Diederen BM, Veenendaal D, Jansen R, Herpers BL, Ligtvoet EE, Ijzerman EP. Rapid antigen test for pandemic (H1N1) 2009 virus. Emerg Infect Dis 16: 897-898, 2010; author reply 898.

6. Amorim VB, Rodrigues RS, Barreto MM, Zanetti G, Hochhegger B, Marchiori E. Influenza A (H1N1) pneumonia: HRCT findings. J Bras Pneumol 39: 323-329, 2013.

7. Mauad T, Hajjar LA, Callegari GD, et al. Lung pathology in fatal novel human influenza A (H1N1) infection. Am J Respir Crit Care 
Med 181: 72-79, 2010.

8. Shieh WJ, Blau DM, Denison AM, et al. 2009 pandemic influenza A (H1N1): pathology and pathogenesis of 100 fatal cases in the United States. Am J Pathol 177: 166-175, 2010.

9. von Ranke FM, Zanetti G, Hochhegger B, Marchiori E. Infectious diseases causing diffuse alveolar hemorrhage in immunocompetent patients: a state-of-the-art review. Lung 191: 9-18, 2013.
10. Quispe-Laime AM, Bracco JD, Barberio PA, et al. H1N1 influenza A virus-associated acute lung injury: response to combination oseltamivir and prolonged corticosteroid treatment. Intensive Care Med 36: 33-41, 2010.

11. Han K, Ma H, An X, et al. Early use of glucocorticoids was a risk factor for critical disease and death from $\mathrm{pH} 1 \mathrm{~N} 1$ infection. Clin Infect Dis 53: 326-333, 2011.

(C) 2015 The Japanese Society of Internal Medicine http://www.naika.or.jp/imonline/index.html 